EMBRYARIDDLE
Aeronautical University

SCHOLARLY COMMONS

\section{International Journal of Aviation,} Aeronautics, and Aerospace

\title{
There is a Bias in Aviation Against Research That is Perceived to be "Easy"
}

\section{Stephen Rice}

ERAU, rices15@erau.edu

Scott R. Winter

Embry-Riddle Aeronautical University, scott.winter@mac.com

Nicola M. O'Toole

Embry-Riddle Aeronautical University, paynen@erau.edu

Follow this and additional works at: https://commons.erau.edu/ijaaa

Part of the Other Psychology Commons

\section{Scholarly Commons Citation}

Rice, S., Winter, S. R., \& O'Toole, N. M. (2019). There is a Bias in Aviation Against Research That is Perceived to be "Easy". International Journal of Aviation, Aeronautics, and Aerospace, 6(4). https://doi.org/10.15394/ijaaa.2019.1357

This Article is brought to you for free and open access by the Journals at Scholarly Commons. It has been accepted for inclusion in International Journal of Aviation, Aeronautics, and Aerospace by an authorized administrator of Scholarly Commons. For more information, please contact commons@erau.edu. 


\section{Introduction}

Research can take on many forms, including various methods and designs. It provides value to the scientific community in the resulting knowledge, which can be used to make informed decisions based on the criteria under investigation. For example, a new policy or procedure can be shared with end-users to determine their willingness to accept or use the desired outcomes. New safety devices can be compared to measure their level of effectiveness. However, within the construct of research, those conducting it must frequently balance tradeoffs such as time to conduct the study, costs related to equipment, compensation amounts to gather a large enough sample, and efficiency to be able to complete the study within the desired time frame. As a result, researchers frequently seek to examine the most efficient and effective way to conduct research studies. However, that can incur the possibility that the readership will view the "scientific contribution" of the study to be lacking somehow.

According to a 2015 Pew Research Center study, $79 \%$ of adults say that science has a positive impact on their lives. The way the public consumes scientific information has changed drastically since the advent of the Internet. Scientific breakthroughs were traditionally communicated by an expert via television, radio or print news sources. The public is now able to browse blogs, Twitter posts, and use a simple Google search to find scientific information of interest to them. The purpose of this current study was to examine possible bias towards research studies which shared the same investigative methods and approaches but only differed on variables related to how easy or difficult it was to conduct the study. A brief definition of research and the scientific method will be provided in the literature review, followed by describing the various types of research, and previous literature related to research rigor, bias and the perceptions of research quality.

\section{Research and the Scientific Method}

\section{Literature Review}

The overall goal of research studies is to contribute to the body of knowledge. To provide a formal definition of research may vary based on discipline, but a general definition states research is "a process through which new knowledge is discovered" (Salkind, 2009, p. 2). This process can be conducted using various methods such as quantitative, qualitative or mixed methods. Additionally, there are a number of designs which can be explored, such as experimental or non-experimental, followed by a series of statistical procedures to produce answers to the research questions (Gall, Gall, \& Borg, 2007).

The selection of these various methods and designs will usually be linked to the research question(s) under investigation. However, these various designs can be influenced by perceptions and biases in viewing some designs as having greater scientific value than others. Qualitative research is frequently perceived as less 
rigorous than quantitative methods (Buckler, 2008; Cawthorne, 2001), but in reality, both methods are rigorous; they are just employed to answer different research questions (Choy, 2014; Kraska, 2008). Their complimentary aspects are further demonstrated in the value of using mixed methods (Edmonds \& Kennedy, 2017; Gall et al., 2007). Therefore, it would be a rather incorrect perception to demonstrate bias toward either method. Similarly, emphasis has been placed on conducting true experiments or randomized controlled trials (RCTs) due to their goal of determining causality (Morrison, 2001) as a result of their ability to provide strong procedural controls and manipulation (Cohen, Manion, \& Morrison, 2000). However, while there are studies where RCTs may be the best option to test certain hypotheses, there are many examples of valuable research, such as gender comparisons, where randomization is not possible within the pre-determined groups. If handled correctly, the penalty to internal validity can be minimized, and the research can still hold value.

An additional aspect related to conducting research is the use of the scientific method. The scientific method is a formal procedure which details the traditional steps in conducting research studies. The steps include forming the research question(s), developing hypotheses, conducting data, testing hypotheses, and allowing for replication (Gall et al., 2007; Salkind, 2009; Wilson \& Joye, 2017). An important aspect of the scientific method is the final step listed-replication. Replication is the step that allows for follow-on research to the initial study. This allows for the results to be examined using different populations or with slight modifications to verify the original findings or expand on the existing body of knowledge. Furthermore, replication can be used, especially within multi-study research projects, to add to the level of rigor of the study design and procedures. Schmidt and Hunter (1995) identified the challenges that can occur to replication if studies are ambiguous in their description of the conceptualization, methods, procedures, and findings. This shortcoming may result in other researchers not being able to complete replication studies and thus restrict further contributions to the cumulative knowledge of the topic under investigation.

\section{Types of Research and Sources of Data}

Research is typically divided into two categories: basic and applied research. Both types of research seek to contribute to the body of knowledge. Basic research may provide contributions which do not have an immediate effect or application, whereas applied research provides more practical and immediate use findings (Salkind, 2009). As a result, there can be a bias shown against basic research as it may not be viewed as immediately contributing something new to the field. However, basic research has been demonstrated under numerous circumstances to provide long-term influences on society (Salkind, 2009), therefore a bias against its usefulness may be misplaced. Even if basic research is never used 
to solve real world problems, there is no reason to question the rigor of the study just because it is not directly applicable to a current problem.

Research studies may also be influenced by the type of data collected for the completion of their studies. Data can be collected from in-person studies such as in a laboratory, through questionnaires deployed either in-person or electronically or through archival sources such as reports and databases (Gall et al., 2007; Wilson \& Joye, 2017). Within the current study, we examine the location/type of data collection, amount of time, and number of researchers involved in the study would influence participant's perceptions on the quality of the study.

These variables represent just some of the tradeoffs that researchers must deal with when conducting research studies. Whether conducting the study with or without funding, there is unlikely to be unlimited financial resources available, and researchers must make decisions about efficiency of time, cost, and management to produce the best quality study given the scarce resources. Factors beyond the complexity of the study itself may affect the variables chosen as a part of this study. As an example, unmerited authorship and ghost writers can overinflate the number of researchers listed, while having no affect on the scientific value of the paper (Shaffer, 2014).

\section{Perceptions on Research Quality}

Perceptions on research quality have also been debated within the scientific community. Some argue that there exists a publication bias from journal editors and reviewers in cases where the strength of the findings and results are significant (Dickerson, 1990; Dwan et al., 2008) over studies where the null effect is retained (Hubbard \& Armstrong, 1997). A 2017 study showed a six percent yearly increase in the proportion of publication of positive results across many countries and scientific disciplines (Mlinarić, 2017). This bias may then prevent the complete dissemination of knowledge to the field, as researchers with null effects may not pursue publication of their studies if they perceive little success in achieving publication. The effect of these perceptions and bias may also lead researchers to conduct studies where significance can be demonstrated, such as through using excessively large samples to result in a $p$-value that achieves significance. The issue of publication bias is considered significant enough that some major organizations and journals have taken a stance. The Committee on Publication Ethics "states that studies with negative results should not be excluded in order to support a debate in science" (Mlinarić, 2017, p. 4).

\section{Rigor}

Mentzer (2008) provided a thorough definition of rigor stating it "is the constant examination of whether research can actually support and justify the 
claims it makes. It implies use of the appropriate theories and methods to avoid concluding something the research did not actually reveal" (p. 72). Gnyawali and Song (2016) suggested three measures of rigor which should be considered in evaluating research studies: 1) Conceptual rigor refers to the theoretical foundation or logic used to understand the construct; 2) methodological rigor relates to how the design of the study provides data to answer the research questions; and 3) empirical describes clearly articulating the data analysis process, procedures, and reporting of results clearly. This definition is important to the current study because when focusing on the rigor, items such as those manipulated within the current study (amount of effort needed to gather participants, amount of time needed to conduct the study, and the number of researchers involved) are clearly not part of what makes a research study rigorous. However, these variables may still influence a person's perception as to the scientific contribution of a research study.

\section{Public Opinion of Scientific Research}

The vast majority of the public sees the value of scientific research. However, when it comes to specific examples of trust, rigor, ethics, and a specific context, the opinions can differ vastly. For example, when it comes to rigor in medical research, the general public and health advocates want rigorous testing to ensure new medications are safe. However, the terminally ill, who may benefit from the medication, would prefer fast tracked testing that may not be as rigorous (Resnik, 2010). The question of how scientifically literate a person has to be has been the subject of much debate. It is generally accepted that a person should have a sufficient breadth of scientific knowledge to allow them to read and understand the material presented in the science section of the New York Times (Miller, 2004). This becomes critically important when a person is making decisions about their own health, or the health of family members. The use of vaccines or lack thereof for example has been featured prominently in the news media; as an example of a health issue that requires a certain level of scientific understanding in order to interpret correctly. This is a substantial challenge for journalists and others seeking to convey the information in a manner that meets the New York Times level of understanding.

With the advent of the Internet the public has direct access to vast quantities of scientific information. The way the information is presented, the types of comments people have posted about the content and the number of "likes" are just a few of the ways that scientific information can be biased when interpreted by the consumer (Brossard, 2013). However, research has also shown that when a member of the public wants to learn more about a scientific subject, they are more likely to seek material that contains information that presents both sides of an argument, or publications that are not opinion based (Brossard, 2013). 
There is significant amount of information regarding how scientific information is communicated and bias; there is little that addresses consumer bias associated with how the research was conducted.

\section{Current Study}

While there have been some prior studies which have explored research rigor and bias, we are not familiar with any prior study in the aviation-related research literature that examines possible bias toward research studies based on the "ease" of which the study was conducted. Therefore, the purpose of the current studies was to examine what variables affect a participant's perceived scientific value of a research study. The effort to gather participants, the time required to complete the study, and the number of researchers involved with the study were manipulated, while holding the actual scenario of the study constant. The effort to gather participants was manipulated by either having the participants readily available in a classroom or by requiring them travel to a laboratory setting. Over a series of four experiments, we predicted the following hypotheses:

$\mathrm{H}_{\mathrm{a} 1}$ : Research that takes less effort, costs less, or requires fewer researchers will be perceived to be easier even though the actual methodology of the studies are identical.

$\mathrm{H}_{\mathrm{a} 2}$ : Perceived "ease/difficulty" of the studies will mediate the relationship between the location of the study, time to conduct the study, and number of researchers involved in the study, and whether or not the research is perceived as being scientific or has meaningful conclusions.

\section{Participants}

\section{Study 1 - Methods}

Four hundred people (229 male/171 female) from the United States took part in the study for compensation through Amazon's Mechanical Turk (MTurk). MTurk provides a method for participants to be compensated for taking surveys. This data has shown to be as reliable as laboratory data and is a useful way of collecting perceptions about aviation research (Buhrmester, Kwang, \& Gosling, 2011; Germine et al., 2012; Rice, Winter, Doherty, \& Milner, 2016). The mean age was $38.02(S D=11.88)$ years.

\section{Materials and Procedure}

Participants first signed an electronic consent form and then read the instructions. Following this, they were presented with the subsequent scenario: "In a recent study, a group of researchers wanted to find out how likely it was for someone to shout for help on an airplane if they saw smoke coming from the engine. They then published the results in an aviation journal." In addition to this, participants were told one of the two following pieces of information; depending on which condition they were randomly assigned to: 
Condition 1: The researchers went to a university class and had participants fill out a questionnaire.

Condition 2: The researchers had participants register for their study, come to their laboratory, and then fill out a questionnaire.

Following this, participants were asked to respond to the following statements on a 5-point Likert scale: 1) This study was scientific (had scientific merit), 2) This study followed rigorous scientific methods, 3) This study was a true experiment, 4) This study was designed correctly, 5) The findings from this study can be generalized, 6) This study was conducted properly, 7) The conclusions from this study will be meaningful, and 8) The journal used for publication was probably a top-tier journal. Lastly, participants provided demographics information, were debriefed, paid, and dismissed.

\section{Study 1 - Results}

Prior to analysis, the data from the scale of eight statements were subjected to a Cronbach's Alpha which revealed extremely high internal consistency (.93) and a Guttmann's Split Half test which revealed extremely high reliability (.92). In addition, a factor analysis using principal component and Varimax rotation indicated that all statements loaded strongly on one factor and explained $69 \%$ of the variance. Therefore, we combined all the scale statements into one average score.

A between-participants $t$-test revealed a significant difference $(p<.05)$ between the two conditions, $t(398)=3.51, p<.001, d=.35$, indicating that the perceived scientific value of the study conducted in the laboratory setting $(M=-$ $0.20, S D=0.92)$ was higher than the one conducted in the classroom $(M=-0.53$, $S D=0.95)$.

\section{Study 1 - Discussion}

The results from Study 1 are straightforward and support the hypothesis that research which takes less effort to collect the data will be perceived to be less scientific even though the actual methodology of the studies is identical. In this case, participants were given a scenario that differed in only one way; either participants were easily collected in the classroom, or they had register and go to a laboratory to fill out the exact same questionnaire.

\section{Study 2 - Introduction}

One might argue that our interpretation of Study 1 is a leap in logic; that is, participants may not have assumed that the study in the classroom was easier than the one done in the laboratory. There may be some other reason for the differences. Therefore, Study 2 was designed to address this limitation. In Study 2, we specifically asked participants how easy/difficult they thought the research was and used that variable as a mediating variable between the type of study and the 
scientific value of the research. We hypothesized that participants would again view the classroom study as less scientific compared to the laboratory study and that this effect would be mediated by perceived ease/difficulty of the research.

\section{Participants}

\section{Study 2 - Methods}

Three hundred and ninety-nine people (208 male/191 female) from the United States took part in the study for compensation through Amazon's Mechanical Turk (MTurk). The mean age of participants was $37.91(S D=12.52)$ years.

\section{Materials and Procedure}

Study 2 was identical to Study 1 with the following exceptions. In addition to the other questions, participants were also asked, "Based on the scenario above, how easy/difficult do you think it was for the researchers to complete the study?" They provided their answers on a scale from 1 (Extremely easy) to 10 (Extremely difficult). This was used as the mediating variable in the analyses. The number of statements for the outcome variable was reduced to two as a result of some participants in Study 1 being confused about the other statements' specific meanings. The statements that remained were: 1) This study was scientific [had scientific merit], and 2) The conclusions from this study will be meaningful.

\section{Study 2 - Results}

A between-participants $t$-test revealed a significant difference $(p<.05)$ between the two conditions, $t(397)=2.20, p=.028, d=.22$, indicating that the perceived scientific value of the study conducted in the laboratory setting $(M=$ $0.20, S D=1.02)$ was higher than the one conducted in the classroom $(M=-0.03$, $S D=1.04)$.

\section{Mediation}

Hayes' (2013) bootstrapping was used to test mediation between the condition and perceived scientific value. Using 5,000 bootstrapped samples, the $95 \%$ confidence interval ranged from .0314 to .1734, indicating significant mediation. Bootstrapping is a random resampling of data to produce a confidence interval. Mathematically 5,000 to 10,000 samples are sufficient to gain the precision needed (Hayes, 2013).

\section{Study 2 - Discussion}

The results of Study 2 replicated those found in Study 1; that is, the participants perceived the classroom study to have less scientific value and to be less meaningful when compared to the laboratory study. In addition, the mediation analysis was significant, providing evidence to support our hypothesis that ease/difficult of the research was the cause of participants viewing the two conditions differently in regard to scientific value. In other words, participants 
viewed the classroom study as less scientific because they perceived it to be easier to conduct.

\section{Study 3 - Introduction}

The data from Studies 1 and 2 revealed that research conducted in the classroom or laboratory would be viewed differentially with regards to scientific merit. Furthermore, it was shown that this result was due to differences in perceived ease/difficulty of conducting the research. In Study 3, we decided to replicate these findings with another manipulation that would again cause participants to view the scientific merit of the study differently depending on perceived ease/difficulty. In Study 3, we manipulated the amount of time it took the researchers to conduct the study. We hypothesized that research taking 100 hours to complete would be perceived as more difficult, and of more scientific merit, than research that took 20 hours to complete.

\section{Participants}

\section{Study 3 - Methods}

Four hundred people (230 male/170 female) from the United States took part in the study for compensation through Amazon's Mechanical Turk (MTurk). The mean age of participants was $36.86(S D=11.16)$ years.

\section{Materials and Procedure}

In Study 3, participants were again presented with a scenario, and then asked, "Based on the scenario above, how easy/difficult do you think it was for the researchers to complete the study?" They provided their answers on a scale from 1 (Extremely easy) to 10 (Extremely difficult). This was used as the mediating variable in the analyses. As in Study 2, the number of statements for the outcome variable was reduced to two. Therefore, Study 3 was identical to Study 2 with the following exception. Instead of manipulating the venue, we manipulated how long the study took to finish. In one condition, the entire study took the researchers about 20 hours to complete. In the other condition, it took them 100 hours to complete. Again, everything else was held constant.

\section{Study 3 - Results}

A between-participants $t$-test revealed a significant difference $(p<.05)$ between the two conditions, $t(398)=2.05, p=.041, d=.21$, indicating that the perceived scientific value of the study that took 100 hours $(M=0.68, S D=0.77)$ was higher than the one that only took 20 hours $(M=0.51, S D=0.89)$.

\section{Mediation}

Hayes' (2013) bootstrapping was used to test mediation between the condition and perceived scientific value. Using 5,000 bootstrapped samples, the 
$95 \%$ confidence interval ranged from .0002 to .0005 , indicating significant mediation.

\section{Study 3 - Discussion}

The data from Study 3 conceptually replicated that of Studies 1 and 2 . Participants who were presented with a study that took 100 hours found that study to be more difficult to complete compared to a study that only took 20 hours to complete. Furthermore, this perception led them to believe that the study had more scientific merit and meaningfulness.

\section{Study 4 - Introduction}

In Study 3, we showed that the amount of time that aviation research takes also has an effect on perceptions of scientific merit. In Study 4, we took this one step further by manipulating how many researchers took part in the study. We hypothesized that research requiring seven researchers to complete would be perceived as more difficult, and of more scientific merit, than research that only required one researcher to complete.

\section{Participants}

\section{Study 4 - Methods}

Six hundred and five people (326 male/279 female) from the United States took part in the study for compensation through Amazon's Mechanical Turk (MTurk). The mean age of participants was $35.67(S D=10.86)$ years.

\section{Materials and Procedure}

In Study 4, participants were again presented with a scenario, and then asked, "Based on the scenario above, how easy/difficult do you think it was for the researchers to complete the study?" They provided their answers on a scale from 1 (Extremely easy) to 10 (Extremely difficult). This was used as the mediating variable in the analyses. As in Studies 2 and 3, the number of statements for the outcome variable was reduced to two. Therefore, Study 4 was identical to Study 3 with the following exception: Instead of manipulating how long the study took to finish, we manipulated how many researchers participated in the study. In one condition, the study only required one researcher to complete it, and in the other condition, it required seven researchers. Again, all other factors were held constant.

\section{Study 4 - Results}

A between-participants $t$-test revealed a significant difference $(p<.05)$ between the two conditions, $t(603)=4.26, p<.001, d=.35$, indicating that the perceived scientific value of the study that required seven researchers $(M=0.64$, $S D=0.83)$ was higher than the one that only required one researcher $(M=0.35, S D$ $=0.88$ ). 


\section{Mediation}

Hayes' (2013) bootstrapping was used to test mediation between the condition and perceived scientific value. Using 5,000 bootstrapped samples, the $95 \%$ confidence interval ranged from .1322 to .2719, indicating significant mediation.

\section{Study 4 - Discussion}

As with Study 1 through 3, the data from Study 4 was straightforward. Research that required seven researchers to complete was viewed as more difficult, and of more scientific merit and meaningfulness, compared to research that only required one researcher to complete. We discuss the implications of these four studies in the general discussion.

\section{General Discussion}

The purpose of this research was to investigate the hypothesis that aviation research that is perceived to be more difficult is also perceived to have more scientific merit and meaningfulness. We note that the only differences between any of the conditions in the four studies was either: 1) the location of where the study was conducted and the amount of effort taken to get participants; 2) the amount of time taken to complete the study; or 3) the number of researchers needed to complete the study. We also note that we only did four studies; that is, there were no other "failed" studies that we are hiding from the reader. In all four studies, the data strongly supported the hypotheses.

In Studies 2-4, we also added in a mediator in order to test the hypothesis that participants perceived the scientific merit of the classroom study, the 20-hour study, and the one-researcher study to be less scientific compared to the corresponding studies because they perceived them to be easier. In all three of these mediation studies, the analyses revealed that ease/difficulty was indeed a significant mediator, and that participants were primarily penalizing the scientific merit of the studies because they perceived them to be easier to conduct (Gnyawali \& Song, 2016). This bias in evaluating the scientific value of the study appears to be solely due to that one variable (Mentzer, 2008).

The findings from all four studies demonstrate a bias against those studies which were perceived to be easier despite no indications that the methodological rigor had changed. This adds to the existing research that demonstrates consumer bias based on how the information is consumed (Bossard, 2013). Additionally, it appears that the actual rigor of the studies, specifically the conceptualization, methods, and empirical results are being overshadowed by variables that do not necessarily have any impact on the level of rigor of the studies conducted (Gnyawali \& Song, 2016).

The findings from the current study support similar findings in the literature related to comparisons in the value of research conducted using quantitative or 
qualitative methods (Buckler, 2008; Cawthorne, 2001; Choy, 2014; Kraska, 2008) and between basic or applied research studies (Gall et al., 2007). Therefore, it is important for both the scientific community, and the consumers of scientific knowledge to be aware of the possible bias towards studies based on how they are conducted. Instead, focus should be on the components related to the level of rigor, namely, the conceptual, methodological, and empirical aspects of the study (Gnyawali \& Song, 2016; Mentzer, 2008).

\section{Practical Applications}

The findings from the current study provide some very practical insights. The data suggests that consumers view research studies that are perceived as "easier" as having less scientific value than those perceived to be more difficult to conduct. These findings expose an apparent bias against otherwise valid studies which contribute knowledge to the body of science. Rather than focusing on the perceived ease in which the study was conducted, focus should be on the methodological rigors and design of the study, which are the decisions that are related to control for threats to internal and external validity. It is these threats which truly effect the validity and generalizability of the results from the study. Value from this current study is provided in identifying this possible bias against studies which are perceived as easier than others with the hopes that in being aware of these either conscious or unconscious thoughts, their effect on assessing the true value of the research contribution can be mitigated.

\section{Limitations}

The current study has some limitations. First, a convenience sample was used from Amazon's Mechanical Turk (MTurk). This provides limits on the generalizability of the findings, and it is restricted to those types of people who complete and participant in online human-subject platforms. Further research should be conducted to replicate this study to other audiences, such as academics and other consumers of scientific research, to see if the findings replicate. The current study was also limited in the number of variables which could be manipulated, and we decided to use the location of the research, amount of time to conduct the study, and number of researchers needed. Future studies should examine other variables to see how the perceived ease of the study could affect additional manipulations. This study uses the term 'ease' as the mediating variable. This is a very subjective word; future studies could attempt to define 'ease' for the participants. Lastly, the data was cross-sectional; that is, collected at one point in time. Follow-on studies could examine these types of issues in a longitudinal format to see if the trends of these results vary over time. 


\section{Conclusions}

The purpose of the current study was to examine how the perceived scientific value of a research study could be manipulated based on the perceived ease to complete the study. In a series of four studies, the data indicates that studies which took less effort to collect data, were shorter to complete, and used fewer researchers, were perceived to have less scientific value compared to studies which took more effort to collect data, took a longer period of time, or required more researchers. Ease/difficultly was shown to be a significant mediator, providing further evidence that perceived ease/difficulty was influencing the results. 


\section{References}

Brossard, D. (2013). New media landscapes and the science information consumer. Proceedings of the National Academy of Sciences, 110(Supplement 3), 14096-14101.

Buckler, K. (2008). The quantitative/qualitative divide revisited: A study of published research, doctoral program curricula, and journal editor perceptions. Journal of Criminal Justice Education, 19(3), 383-403.

Buhrmester, M., Kwang, T., \& Gosling, S. D. (2011). Amazon's Mechanical Turk: A new source of inexpensive, yet high-quality data? Perspectives on Psychological Science, 6(3), 3-5.

Cawthorne, P. (2001). Identity, values, and method: Taking interview research seriously in political economy. Qualitative Research, 1, 65-90.

Cohen, L., Manion, L., \& Morrison, K. R. B. (2000). Research methods in education (5th ed.). London: Routledge.

Choy, L. T. (2014). The strengths and weaknesses of research methodology: Comparison and complimentary between qualitative and quantitative approaches. IOSR Journal of Humanities and Social Science, 19(4), 99104.

Dickersin, K. (1990). The existence of publication bias and risk factors for its occurrence. Journal of the American Medical Association, 263(10), 13851389.

Dwan, K., Altman, D. G., Arnaiz, J. A., Bloom, J., Chan, A., Cronin, E., .. . Williamson, P. R. (2008). Systematic review of the empirical evidence of study publication bias and outcome reporting bias. PLOS ONE, 3(8), 1-31.

Edmonds, W. A. \& Kennedy, T. D. (2017). An applied guide to research designs: Quantitative, qualitative, and mixed methods. Thousand Oaks, CA: Sage.

Gall, M. D., Gall, J. P., \& Borg, W. R. (2007). Educational research: An introduction (8th ed.). New York, NY: Pearson.

Germine, L., Nakayama, K., Duchaine, B. C., Chabris, C. F., Chatterjee, G., \& Wilmer, J. B. (2012) Is the web as good as the lab? Comparable performance from web and lab in cognitive/perceptual experiments. Psychonomic Bulletin \& Review, 19(5), 847-857.

Gnyawali, D. R., \& Song, Y. (2016). Pursuit of rigor in research: Illustration from coopetition literature. Industrial Marketing Management, 57, 12-22.

Hayes, A. F. (2013). Introduction to mediation, moderation, and conditional process analysis: A regression-based approach. New York, NY: Guilford Press.

Hubbard, R., \& Armstrong, J. S. (1997). Publication bias against null results. Psychological Reports, 80, 337-338.

Kraska, P. B. (2008). The third way: Teaching mixed methods research. ACJS Today, 23(1), 1-8. 
Mentzer, J. T. (2008). Rigor versus relevance: Why would we choose only one? Journal of Supply Chain Management, 44(2), 72-77.

Miller, J. D. (2004). Public understanding of, and attitudes toward, scientific research: what we know and what we need to know. Public Understanding of Science, 13 273-294.

Mlinarić, A., Horvat, M., \& Šupak Smolčić, V. (2017). Dealing with the positive publication bias: Why you should really publish your negative results. Biochemia medica, 27(3), 1-6.

Morrison, K. (2001). Randomised controlled trials for evidence-based education: Some problems in judging 'what works.' Evaluation \& Research in Education, 15(2), 69-83.

Pew Research Center (2015, January 2015). Opinion differences between public and scientists. Retrieved from https://www.pewinternet.org/2015/01/29/public-and-scientists-views-onscience-and-society/pi_2015-01-29_science-and-society-00-01/

Resnik, D. B. (2011). Scientific research and the public trust. Science and engineering ethics, 17(3), 399-409.

Rice, S., Winter, S. R., Doherty, S. \& Milner, M. N. (2017). Advantages and disadvantages of using internet-based survey methods in aviation-related research. Journal of Aviation Technology and Engineering, 7(1), 58-65.

Salkind, N. (2009). Exploring research (7th ed.). Upper Saddle River, NJ: Pearson Prentice Hall.

Schmidt, F., \& Hunter, J. E. (1995). The impact of data-analysis methods on cumulative research knowledge statistical significance testing, confidence intervals, and meta-analysis. Evaluation and the Health Professions, 18(4), 408-427.

Shaffer, E. (2014). Too many authors spoil the credit. Canadian Journal of Gastroenterology \& Herpetology, 28(11), 605.

Wilson, J. H. \& Joye, S. W. (2017). Research methods and statistics: An integrated approach. Thousand Oaks, CA: Sage. 